\title{
Reacciones adversas a medicamentos en el servicio de medicina interna del Hospital Universitario de Neiva
}

\section{Adverse Drug Reactions Occurring in the Internal Medicine Service at University Hospital in Neiva}

\author{
Cindy Lorena Beltrán Endoํㅡ, Giovanni Caviedes Perez². \\ 1 Residente Medicina Interna. Departamento de Medicina Interna. Hospital Universitario Her- \\ nando Moncaleano Perdomo. \\ 2 Médico Internista, Farmacólogo Clínico, Epidemiólogo. Docente, Universidad Surcolombiana. \\ Correspondencia: Giovanni Caviedes Pérez, Correo electrónico: giocape@hotmail.com
}

Fecha de aceptación: Septiembre 15-2013

Beltrán C, Caviedes G. Adverse Drug Reactions Occurring in the Internal Medicine Service at University Hospital in Neiva. RFS Revista Facultad de Salud. 2013; 5 (2): 10-17

\section{Resumen}

Objetivo: Establecer la prevalencia de las reacciones adversas a medicamentos que ocurren en el servicio de Medicina Interna del Hospital Universitario de Neiva.

Diseño: Descriptivo de tipo corte transversal

Lugar: Hospital Universitario Hernando Moncaleano Perdomo (HMP), Neiva- Colombia.

Población: pacientes hospitalizados en el servicio de Medicina Interna del Hospital Universitario de Neiva Hernando Moncaleano Perdomo.

Método: Recolección de datos con observación directa no participativa, siguiendo un modelo de farmacovigilancia activa. Se realizó la revisión documental de las historias clínicas.

Resultados: De 303 pacientes hospitalizados en el servicio se obtuvieron 153 pacientes que presentaron RAM que correspondieron al $50 \%$ de la muestra. En los que la media de edad fue $63+/-4,6$. Las reacciones adversas a medicamentos (RAM) se agruparon por sistemas: el 27,4\% afectó la piel, el $13,8 \%$ el sistema gastrointestinal, seguidos de sistema hematológico y cardíaco. Con respecto a los medicamentos relacionados con las RAM se dividieron en grupos: antimicrobianos en un 37,9\%, seguido de antihipertensivos con un 17,3\%, anticoagulantes en un $9,8 \%$. Los tres medicamentos más frecuentes en su orden: vancomicina en un 6,6\%, el enalaprilen un 6,6\% y tramadol con un 5,3\%. Al clasificar las RAM se encontró que el 53,6\% correspondieron al tipo A y $46,4 \%$ al tipo B. Estas, representaron 71 casos del total, las más frecuentes fueron exantema en 39,4\%, prurito 14,3\%, la tos 11,2\% y se presentó un caso de DRESS (Drug Reaction With Eosinophilia and Systemic Symptoms Syndrome). Se documentaron 14 casos de RAM graves representadas en sangrado 35,8\%, IRA en un $35,8 \%$ entre otras. Los medicamentos relacionados con estas RAM fueron: warfarina $14,4 \%$, igual que enoxaparina y la vancomicina. 
Palabras clave: reacciones adversas a medicamentos, farmacovigilancia.

\begin{abstract}
Objective: to establish the prevalence of adverse drug reactions (ADR) occurring in the internal medicine service at the Hospital Universitario in Neiva.

Design: cross-sectional, descriptive study.

Place: Hospital Universitario Hernando Moncaleano Perdomo, Neiva-Colombia

Population: patients in Internal Medicine service of Hospital Universitario Hernando Moncaleano Perdomo.

Method: Data collection with non-participative direct observation, following an active pharmaco surveillance model. Documentary review of medical histories was performed.

Results: of 303 inpatients in the internal medicine service it was obtained 153 patients with ADRs corresponding to $50 \%$ of the sample. Their average age was $63+/-4,6$. ADRs were grouped by systems: $27.4 \%$ affected skin, $13.8 \%$ affected the gastrointestinal system, followed by the hematologic and cardiac systems. Regarding medicines related to ADRs, they were divided into groups, as follows: antimicrobials by $37,9 \%$, followed by antihypertensives by $13.3 \%$, anticoagulants by $9,8 \%$. The three most frequent drugs were: vancomycin by $6.6 \%$, enalaprilen by $6,6 \%$ and tramadol by $5.3 \%$. When classifying ADRs it was found that $63 \%$ corresponded to type A and $46,4 \%$ to type B. These ADRs represented 71 cases of the total; the most frequent were: rash by $39.4 \%$, pruritus by $14.3 \%$ and cough by $11.2 \%$ and a Drug Reaction With Eosinophilia and Systemic Symptoms Syndrome (DRESS) case took place. Fourteen severe cases of ADRs were documented which showed bleeding by 35,8\%, IRA by $35,8 \%$ among others. Drugs related to these ADRs were: warfarin by $14.4 \%$ as well as enoxaparin, and vancomycin
\end{abstract}

Keywords: adverse reactions to medicine, pharmaco surveillance.

\section{Introducción}

Las reacciones adversas a medicamentos (RAM), son una respuesta nociva del organismo desencadenada por el uso de un fármaco a dosis terapéuticas provocando síntomas que pueden ser de leves a severos ${ }^{(1)}$. Estas reacciones se han relacionado con el aumento en la hospitalización, mayor morbilidad y mortalidad. Las RAM constituyen un problema de salud pública ya que alrededor del 2-5\% de todas las hospitalizaciones se atribuyen a algún tipo de reacción adversa, y el $20 \%$ de los pacientes hospitalizados pueden presentarla ${ }^{(2)}$; es una condición que, disminuye la calidad de vida de los pacientes, ocasiona perdida de la confianza en los médicos, y eleva el costo de la atención.

Las RAM se clasifican de acuerdo a su causalidad, a su mecanismo de producción y a su seve$\operatorname{ridad}^{(3)}$. De acuerdo a su causalidad se utilizan varios métodos para establecer la imputabilidad de la misma; uno de los más usados es el algorítmo de naranjo, donde por medio de una serie de preguntas se establece una puntuación y se considera si un evento dado es una RAM posible, dudosa, probable o probada.

De acuerdo a su mecanismo de producción se clasifican en A, B, C, D, E y F. Las más reconocidas son la A y la B. Las RAM tipo A consisten en una extensión del efecto farmacológico y dependen de la dosis; las tipo B son las llamadas idiosincráticas ya que su mecanismo de producción es desconocido. De acuerdo a su severidad son leves, moderadas, graves y mortales.

En un estudio realizado por Lazarou et al. reportaron que la incidencia de RAM en EEUU es cerca de 2 millones anuales con una mortalidad del $0.12-0.3 \%$, aumentando la estancia intrahospitalaria de los pacientes y los gastos de la enti- 
dad de salud. ${ }^{(4)}$ Martín et al.; describió en su estudio la aparición de RAM en el 26,69\% de 377 pacientes hospitalizados en el centro médico de México; los medicamentos referidos como primera causa fueron los $\beta$-lactamicos y antineoplásicos. ${ }^{(5)}$ En Colombia, Triviño et al. reportó un incidencia de $25,1 \%$ de eventos adversos en un hospital de tercer nivel en Bogotá( ${ }^{(6)}$ y en la ciudad de Cali, López et al., en su estudio describe la aparición de RAM en el 45\% de los pacientes hospitalizados ${ }^{(7)}$.

Por otro lado el efecto del medicamento depende de distintos factores, la molécula del fárma$\mathrm{co}$, la genética del individuo, la inmunogenicidad, el estado metabólico, la célula blanco, la presencia o no de otras patologías. Por esto, luego de fabricado el medicamento y pasado por las fases de prueba, se debe seguir la vigilancia de estos compuestos ${ }^{(8-11)}$.

Viendo así todos estos aspectos relacionados con el uso de medicamentos nace la Farmacovigilan$\mathrm{cia}^{(9)}$. En nuestro medio no se aplica de manera adecuada; no tenemos información epidemiológica que permita conocer las reacciones adversas, que medicamentos se relacionan; por esta razón en este estudio se describen las reacciones adversas a medicamentos del servicio de medicina interna del Hospital Universitario de Neiva, para así crear una pauta, informando e incentivando un mejor uso de los medicamentos.

\section{Metodología}

Diseño del estudio: Es un estudio descriptivo de corte transversal prospectivo.

Población: pacientes hospitalizados en el servicio de medicina interna del Hospital Universitario de Neiva Hernando Moncaleano Perdomo y que cumplan con los siguientes criterios de inclusión y exclusión.

\section{Criterios de inclusión:}

1. Pacientes que presenten signos, síntomas y hallazgos de laboratorios compatibles con reacción adversa asociada a medicamentos.
2. Hospitalizados en el servicio de Medicina Interna, urgencias, piso y UCI.

\section{Criterios de exclusión:}

1. Pacientes con laboratorios tomados de otras instituciones.

Tamaño de la muestra: De un total de 303 pacientes hospitalizados en el servicio de medicina interna, con un nivel de confianza del 95\%, una precisión del $5 \%$ y una proporción del evento tomada de la literatura de un $24-25 \%{ }^{(5-6)}$, se obtuvo una muestra de 153 pacientes, estudio realizado en un periodo de tiempo de 4 meses.

Técnicas de recolección: se obtuvieron los datos mediante la observación directa no participativa, siguiendo un modelo de farmacovigilancia activa. Se hizo una revisión documental de las historias clínicas en un periodo de 4 meses, enero-abril del año 2012; registrando los datos de remisión e ingreso en un formulario que contiene datos epidemiológicos, demográficos y sociales. Se realizó diariamente una visita para la identificación, registro y clasificación de las distintas reacciones adversas presentadas. Se usó el algoritmo de naranjo para establecer la causalidad.

Control de sesgos: Para el sesgo de selección las personas encargadas de la recolección fueron las mismas, manejando un mismo concepto; además se diseñó un cuestionario con preguntas claras. Para el sesgo de medición, se aplicó la escala de naranjo para establecer la causalidad y se excluyeron los paraclínicos provenientes de otras instituciones.

Análisis estadístico: los datos fueron recolectados en una hoja de excel y luego analizados en el paquete estadístico epi-info versión 7.0. Se realizó una estadística descriptiva con la obtención de medidas de tendencia central y de dispersión.

Consideraciones éticas: de acuerdo a la resolución 8430 este estudio es considerado de bajo riesgo. 


\section{Resultados}

Del total de pacientes, 303 en total el 50\% correspondiente a 153 pacientes presentaron algún tipo de reacción adversa; de los cualesel $63,4 \%$ correspondían al servicio de urgencias; el $57,5 \%$ de los casos fueron hombres y el $66 \%$ provenían de área urbana. La media de la edad fue63 +/- 4,6. Las RAM presentadas por los pacientes, se agruparon por sistemas; de estas el $27,4 \%$ corresponde a piel, seguido del gastrointestinal con un $13,8 \%$, del sistema hematológico y cardíaco en un $13,2 \%$ cada uno (Tabla 1 ).

Tabla 1: Reacciones Adversas a medicamentos agrupadas por sistémas en los pacientes de Medicina Interna

\begin{tabular}{lc}
\hline \multicolumn{1}{c}{ Sistema } & $\mathbf{n}(\mathbf{\%})$ \\
\hline Piel & $42(27,4)$ \\
Renal & $6(3,9)$ \\
Respiratorio & $10(6,5)$ \\
Páncreas & $1(0,6)$ \\
Hematológico & $20(13,2)$ \\
Cardiaco & $20(13,2)$ \\
Hígado & $3(1,9)$ \\
Neurológico & $10(6,5)$ \\
Psiquiátricos & $9(5,8)$ \\
Metabólico & $11(7,2)$ \\
Gastrointestinales & $21(13,8)$ \\
\hline TOTAL & $153(100)$ \\
\hline
\end{tabular}

Al clasificar las reacciones adversas de acuerdo a su mecanismo generador se encontró que el $53,6 \%$ correspondían al tipo A y el $46,4 \%$ al tipo B; no se presentaron otros tipos de RAM. De acuerdo a su causalidad aplicando el algoritmo de naranjo podemos decir que el $58,2 \%$ de las RAM fueron probables, el $32 \%$ fueron posibles, el $9,2 \%$ probadas y un caso fue dudosa (Tabla 2 ).

Tabla 2: Clasificación de las Reacciones Adversas a Medicamentos de acuerdo a su causalidad

\begin{tabular}{lc}
\hline \multicolumn{1}{c}{ Naranjo } & n (\%) \\
\hline Probada & $14(9,2)$ \\
Probable & $89(58,2)$ \\
Posible & $49(32)$ \\
Dudosa & $1(0,6)$ \\
\hline TOTAL & $153(100)$ \\
\hline
\end{tabular}

La severidad de las RAM se clasificó de leve a grave, los resultados nos muestran que el 53,6\% fueron leves, el 37,2\% moderadas y el 9,2\% graves. Ninguna fue mortal (Tabla 3).

Tabla 3: Clasificación de las Reacciones Adversas a Medicamentos de acuerdo a su severidad

\begin{tabular}{lc}
\hline \multicolumn{1}{c}{ Severidad } & n (\%) \\
\hline Leve & $82(53,6)$ \\
Moderada & $57(37,2)$ \\
Grave & $14(9,2)$ \\
Mortal & $0(0)$ \\
\hline TOTAL & $153(100)$ \\
\hline
\end{tabular}

Con respecto a los medicamentos relacionados se ordenaron de acuerdo al grupo terapéutico y se encontró que los más frecuentes fueron los del grupo de antimicrobianos con un 37,9\%, seguido de antihipertensivos con un 17,3\%, anticoagulantes en un $9,8 \%$, psicofármacos en un $7,8 \%$, opioides en un 7,2\% y antiinflamatorios no esteroideos en un 3,9\% (Tabla 4).

Tabla 4: Grupo de medicamentos relacionados con las reacciones adversas en los pacientes del servicio de Medicina Interna

\begin{tabular}{lc}
\hline Grupo farmacológico & $\mathbf{n}(\mathbf{\%})$ \\
\hline Antimicrobiano & $58(37,9)$ \\
Antihipertensivos & $26(17,3)$ \\
Anticoagulante & $15(9,8)$ \\
Psiquiátricos & $12(7,8)$ \\
Opioides & $11(7,2)$ \\
Aines & $6(3,9)$ \\
Hipoglucemiantes & $4(2,6)$ \\
Esteroides & $4(2,6)$ \\
Contraste & $2(1,3)$ \\
Broncodilatador & $3(1,9)$ \\
Ablandador & $3(1,9)$ \\
Anticonvulsivante & $3(1,9)$ \\
Inmunosupresor & $2(1,3)$ \\
Anticolinérgico & $2(1,3)$ \\
Adrenérgico & $2(1,3)$ \\
\hline TOTAL & $153(100)$ \\
\hline
\end{tabular}


Los medicamentos más frecuentes en su orden fueron la vancomicina en un $6,6 \%$, el enalapril en un $6,6 \%$, el tramadol con un 5,3\%, el haloperidol y la enoxaparina con un $3,9 \%$ cada una y la dipirona en un 3,3\% (Tabla 5).

Tabla 5: Medicamentos relacionados con las Reacciones Adversas en los pacientes del servicio de Medicina Interna

\begin{tabular}{lc}
\hline \multicolumn{1}{c}{$\begin{array}{c}\text { Medicamentos más } \\
\text { frecuentes }\end{array}$} & n (\%) \\
\hline Vancomicina & $10(6,6)$ \\
Enalapril & $10(6,6)$ \\
Tramadol & $8(5,3)$ \\
Haloperidol & $6(3,9)$ \\
Enoxaparina & $6(3,9)$ \\
Dipirona & $5(3,3)$ \\
Heparina & $4(2,6)$ \\
Cefazolina & $4(2,6)$ \\
Ampicilina- sulbactam & $4(2,6)$ \\
Insulina & $3(1,9)$ \\
Otros & $93(60,7)$ \\
\hline TOTAL & $153(100)$ \\
\hline
\end{tabular}

Las RAM de tipo B corresponden a un porcentaje importante con 71 casos del total. De estas el rash corresponde a un 39,4\% de los casos, seguido del prurito en un $14,3 \%$, la tos en un $11,2 \%$. Se presentó un caso de DRESS (Tabla 6).

Tabla 6: Reacciones adversas a medicamentos clasificadas como tipo B en los pacientes del servicio de Medicina Interna

\begin{tabular}{lc}
\hline \multicolumn{1}{c}{ RAM tipo B } & n (\%) \\
\hline Rash & $28(39,4)$ \\
Prurito & $10(14,3)$ \\
Tos & $8(11,2)$ \\
flebitis química & $7(9,8)$ \\
IRA & $4(5,6)$ \\
Dress & $1(1,4)$ \\
Otros & $13(18,3)$ \\
\hline TOTAL & $71(100)$ \\
\hline
\end{tabular}

De los 14 casos de RAM graves, el sangrado corresponde a un 35,8\%, insuficiencia renal aguda a un $35,8 \%$. Otros de menor frecuencia como la hipoglucemia, la hepatitis, el DRESS y la pancreatitis también fueron evidenciados (Tabla 7). Los medicamentos que más frecuentemente se relacionaron con RAM graves fueron la warfarina, la enoxaparina y la vancomicina en un $14,4 \%$ cada una y en menor medida aparece la heparina, la fenitoína, el ganciclovir y el Iopamiron (medio de contraste) (Tabla 8).

Tabla 7: Reacciones Adversas a Medicamentos graves en los pacientes del servicio de Medicina Interna

\begin{tabular}{lc}
\hline Reacciones adversas graves & $\mathbf{n}(\%)$ \\
\hline Sangrado & $5(35,8)$ \\
IRA & $5(35,8)$ \\
Hipoglucemia & $1(7,1)$ \\
Hepatitis & $1(7,1)$ \\
Dress & $1(7,1)$ \\
Pancreatitis & $1(7,1)$ \\
\hline TOTAL & $14(100)$ \\
\hline
\end{tabular}

Tabla 8: Medicamentos relacionados con las reacciones adversas graves de los pacientes del servicio de Medicina Interna

\begin{tabular}{lc}
\hline \multicolumn{1}{c}{ Medicamentos } & n (\%) \\
\hline Warfarina & $2(14,4)$ \\
Enoxaparina & $2(14,4)$ \\
Vancomicina & $2(14,4)$ \\
Heparina & $1(7,1)$ \\
Fenitoina & $1(7,1)$ \\
Ganciclovir & $1(7,1)$ \\
Iopamiron & $1(7,1)$ \\
Anfotericina B & $1(7,1)$ \\
Glucantime & $1(7,1)$ \\
Insulina & $1(7,1)$ \\
Rifampicina & $1(7,1)$ \\
\hline TOTAL & $14(100)$ \\
\hline
\end{tabular}




\section{Discusión}

En el estudio la frecuencia de las RAM fue del $50 \%$, más alto que lo descrito en la literatura anteriormente citada, quizás por la falta de un programa de farmacovigilancia que vigile y controle estos eventos, que busque de manera diaria la presencia o no de RAM. Los pacientes mayores de 45 años fueron los que presentaron más RAM , donde $28,1 \%$ tenían entre $46-60$ años, el 22,3\% de 61-75 años, con una edad media de $63+/-4,6$. Dato que corresponde con la literatura, según Ocampo y cols. ${ }^{(9)}$ las RAM se presentan con mayor frecuencia en pacientes de edad avanzada, esta constituye un factor de riesgo importante para la aparición de RAM, ya que los pacientes de este grupo etáreo disminuyen la reserva funcional, produce cambios farmacodinámicos y farmacocinéticas, tienen múltiples comorbilidades y están expuestos a polifarmacia ${ }^{(12)}$. La distribución por género es similar al estudio de Ocampo donde las RAM se presentaron un 50\% en hombres.

Danza A y cols. ${ }^{(13)}$ en el 2010, encontraron que los sistemas más comprometidos por RAM fueron el gastrointestinal (48.1\%) y metabólico $(37 \%)$, sin embargo Hafnerw et al. ${ }^{11}$ encontraron unas series que incluyen el cardiovascular en segundo lugar. En nuestro estudio el 27,4\% de las RAM correspondieron a piel, el segundo sistema más frecuentemente afectado fue el gastrointestinal con un $13,8 \%$, seguidos de sistema hematológico y cardíaco, condición que se correlaciona con la mayor frecuencia de reacciones de tipo $B$, secundarias posiblemente a respuestas inmunológicas cuyo principal blanco es el tegumento.

Al clasificar las reacciones adversas encontramos que el 53,6\% corresponden al tipo A y el $46,4 \%$ del tipo B, hecho que concuerda en orden de frecuencia pero difiere en porcentaje de presentación con lo descrito por López y cols. ${ }^{15}$ en su estudio, mostraron que el $92 \%$ de las RAM fueron tipo A (dependiente de la dosis, previsible y común) y el $8 \%$ restante fueron clasifi- cadas como tipo B (independientes de la dosis, impredecibles, poco frecuentes). Las de tipo A siguen siendo las más frecuentes pero en nuestra serie de casos la frecuencia de RAM tipo B es mayor comparado con el estudio de López; esta situación podría posiblemente deberse a un menor control farmacológico, mayor polifarmacia e interacciones medicamentos asa, además de la susceptibilidad genética. Con respecto a las RAM tipo B, el mismo autor describe que la mayoría corresponden a erupción Maculopapular, datos que se correlacionan con nuestro estudio en el que encontramos que el Rashen un 39,4\% y el prurito en un $14,3 \%$ son las más frecuentes de las RAM de tipo $B^{(15)}$.

La causalidad obtenida mediante la escala de naranjo muestra que el 58,2\% de las RAM fueron probables, el $32 \%$ fueron posibles, el 9,2\% probadas y un caso fue dudosa. La frecuencia de las probables y posibles son similares con el estudio realizado por López y cols. ${ }^{(15)}$, donde de un total de 99 pacientes las probables fueron 29 , las posibles 21 y las dudosas 49 .Ninguno de los eventos adversos se clasificaron como definitivas. Nuestro estudio tuvo una frecuencia de RAM probables y posibles más alta; esto quizás se deba, que al tener mayores RAM de piel, lo más probable es que sea de origen medicamentoso y no de la patología de base.

Los medicamentos relacionados con las RAM fueron los antimicrobianos, seguido de antihipertensivos y anticoagulantes. Este resultado es concordante con el estudio de Danza et al. ${ }^{(13)}$ donde sus medicamento más frecuentemente implicados los fueron antimicrobianos y cardiovasculares. La principal causa de hospitalización de nuestra población por demografía es la infecciosa y la cardiovascular, lo que aumenta la exposición de nuestros pacientes a este tipo de medicamentos.

La presencia de sangrado como manifestación de RAM grave se debe al uso de medicamentos anticoagulantes en pacientes de edad avanzada que están expuestos por sus comorbilidades a 
la polifarmacia, generando interacciones medicamentosas; además la reacción adversa se ve posiblemente potenciada por susceptibilidad genética y alteración del metabolismo enzimático de cada paciente. La warfarina es un anticoagulante usado frecuentemente que tiene un comportamiento anticoagulante impredecible, asociado a las múltiples interacciones medicamentosas ocasionadas por las enzimas metabolizadoras como CYP 2C9 pueden potenciar el efecto de la warfarina, visto con los aines, antibióticos como ciprofloxacina, trimetropim y la amiodarona $^{(16) .}$

Por otro lado la insuficiencia renal aguda está ligada al uso de medicamentos nefrotóxicos como ganciclovir, vancomicina, medios de contaste, la necesidad de utilizarlos sin ajustarlos a la función renal previa, a los medicamentos usados conmomitantemente, la falta de evaluación del número de medicamentos nefrotoxicos usados en un mismo paciente pueden generar este tipo de complicaciones catastróficas ya que aumenta la mortalidad y reduce la sobrevida.

Las reacciones adversas a medicamentos son una condición asociada a la terapéutica, por tal motivo las políticas de farmacovigilancia están orientadas al control de éstas y a la disminución de RAM prevenibles. Políticas que deberíamos empezar a implementar por medio de la vigilancia el control de las interacciones y el ajuste de las dosis de los medicamentos. Se recomienda implementar el sistema de farmacovigilancia activa en la institución.

\section{Conclusiones}

Las reacciones adversas más frecuentes fueron las que comprometían piel seguidos de las RAM con compromiso hematológico y cardíaco. Los antimicrobianos, antihipertensivos y los anticoagulantes fueron los 3 grupos de medicamentos más frecuentes en nuestro estudio. Los medicamentos relacionados con RAM graves fueron la warfarina, la enoxaparina y la vacomicina.

\section{Agradecimientos}

Agradecemos al Hospital Universitario de Neiva, y al Programa de Medicina Interna de la Universidad Surcolombiana.

\section{Bibliografía}

1. INCH J, WATSON MC. Patient vesus healthcare professional spontaneous adverse drug reaction reporting. A systematic review. Drugs safe2012; 35 (10): 807-818.

2. TABORA L, AMARAL B. Drugs induced vasculitis. Adverse drug reaction bulletin. April 2013. 279 (1): 1075-1078.

3. KAVITHA D. Adverse drug reaction monitoring pharmacovigilance. J Pharm Res Health Care 2010, pag:127-134.

4. LAZAROU J, POMERANZ BH. Incidence of adverse drug reactions in hospitalized patients: a meta-analysis of prospective studies. Jama 1998;pag: 1200-1205.

5. BECERRIL A. Encuestade reacciones adversas amedicamentos en pacientes hospitalizados. Martín. Rev Alergia Mex 2011;58(4):179-184.

6. TRIBINO G. Direct costs and clinical aspects of adverse drug reactions in patients admitted to a level 3 hospital internal medicine ward. Biomedica. 2006; 26: 31-41.

7. LÓPEZ et al; Adverse drug reactions in internal medicine units at a university hospital: A descriptive pilot study. Colomb Med. 2010; 41: 45-51.

8. KELLY W. Potential risks and prevention: Part 1. fatal adverse drug events. Am J Health. Sys Pharm 2001, pag:1317-24.

9. Pharmacovigilance: ensuring the safe use of medicines. WHO Policy Perspectives on Medicines. World Health Organization 2004 . pg 1-5. January 26, 2010.

10. World Health Organization -WHO- collaborating center for international drug monitoring. The importance of Pharmacovigilance. 2002.

11. ANNIE P. C. Data-driven Methods to Discover Molecular Determinants of Serious Adverse Drug Events. Clin Pharmacol Ther. 2009 March; pag: 259-268. 
12. OCAMPO JM. Aspectos farmacológicos y reacciones adversas medicamentosas. En: López JH, Cano CA, Gómez JF (eds). Fundamentos de medicina: Geriatría. Bogotá: Corporación para Investigaciones Biológicas; 2006. p. 128-32.

13. DANZA A. Adverse drug reaction in an internal medicine unit at the University Hospital. Fonte: Rev. médica Urug; 26(3): 138-144, set. 2010.

14. HAFNER JW, BELKNAP SM. Adverse drug events in emergency department patients. Ann Emerg Med. 2002; 39: 258-67.
15. LÓPEZ LC, col et. Reacciones adversas a medicamentos en las unidades de medicina interna en un hospital universitario: un estudio piloto descriptivo. Colomb. Med. vol.41 no.1 Cali. 2010

16. HOWARD PA, ELLERBECK EF. The nature and frequency of potential warfarin drug interactions that increase the risk of bleeding in patients with atrial fibrillation. Pharmacoepidemiol Drug Saf 2002;pag:569-76. 